\title{
Contracts versus Salaries in Matching
}

\author{
By FEDERICO ECHENIQUE*
}

Workers and firms may bargain over general, multidimensional contracts; they may negotiate over health benefits, housing, retirement plans, etc. Substitutes, on the other hand, is the assumption commonly placed on firms' preferences to guarantee the existence of stable matchings of workers and firms. In this paper I show that, when firms regard contracts as substitutes, bargaining over contracts can be embedded into a model of bargaining over wages.

The economics of the embedding is straightforward, except for a small twist. When a firm and a worker negotiate over a contract, they may bargain over many dimensions. However, the Pareto frontier of contracts is, in a sense, "one dimensional": what is better for the worker is worse for the firm. So Pareto optimal contracts may be viewed as salaries, with the better contracts for the firm meaning lower salaries, and the better contracts for the worker meaning higher salaries. The twist is that a firm's ranking over contracts might be affected by the firm's other hires. For example, health plan A may be better than B for a firm if it has many employees, but B beats A if it has few. When contracts are substitutes, it turns out that the ranking is not affected in this way.

Hatfield and Milgrom (2005) present a model of two-sided worker-firm matching with contracts. A firm will hire a collection of workers and will negotiate a contract with each one of them. The model is a generalization of Kelso and Crawford (1982), where each firm and each worker negotiate a wage. ${ }^{1}$ Kelso and Crawford show that, when firms' demands satisfy gross substitutes, the core of the matching market is nonempty. Hatfield and Milgrom show that, when the firms' preferences over contracts satisfy their notion of substitutes, the core of the market is nonempty.

I show that Hatfield and Milgrom's model can be embedded into the model of Kelso and Crawford. Hatfield and Milgrom's assumption of substitutability enables an embedding where firms' demands for workers satisfy Kelso and Crawford's notion of gross substitutes. As a result, the nonemptiness of the core follows from the argument in Kelso and Crawford, and their salary adjustment algorithm finds a stable matching of workers to firms, and a vector of supporting salaries.

Hatfield and Milgrom's paper is an elegant analysis of two-sided matching. It simplifies matching models and makes their relationship to auction models more transparent. Their paper contributes much more than showing the nonemptiness of the core when firms and workers can sign general contracts, and my result does not diminish

\footnotetext{
* Division of the Humanities and Social Sciences, California Institute of Technology, Pasadena, CA 91125 (e-mail: fede@caltech.edu). I thank Vince Crawford, Flip Klijn, Scott Kominers, and Michael Ostrovsky for very useful comments. I am also very grateful to three anonymous referees for their thoughtful questions and suggestions.

† To view additional materials, visit the article page at http://dx.doi.org/10.1257/aer.102.1.594

${ }^{1}$ Kelso and Crawford build on the analysis of Crawford and Knoer (1981); see Roth and Sotomayor (1990) for a description of the models.
} 
their contribution in the least. I believe, however, that there is value in clarifying the relationship between contracts and salaries. One step in that direction is taken by Hatfield and Kojima (2010), who investigate conditions on preferences over contracts that are weaker than substitutes and still generate stable matchings. My embedding does not work under Hatfield and Kojima's weaker conditions (see Section IIC below).

Future research should explain the consequences of the added generality of contracts over salaries in different economic environments. A paper that seeks to extend some classical result on matching to matching with contracts would need to sort out to what extent allowing for contracts provides a more general result. It may be that contracts are not more general, which would not by itself invalidate the exercise but would be an important feature to understand.

\section{Embedding}

\section{A. Definitions}

I shall describe two models. The model of a matching market with contracts with substitutable choices is due to Hatfield and Milgrom (2005). The model of a matching market with salaries and gross substitutes in demand is due to Kelso and Crawford (1982).

Contracts.-A matching market with contracts is described by:

- (finite, disjoint) sets $W$ of workers, $F$ of firms, and $X$ of contracts; each contract $x \in X$ is assigned one worker $x_{W} \in W$ and one firm $x_{F} \in F$

- for each worker $w \in W$, a utility function $u_{w}: X \cup\{\emptyset\} \rightarrow \Re$; and for each firm $f \in F$, a utility function $u_{f}: 2^{X} \rightarrow \Re$; all utility functions are one-to-one (preferences are strict).

A firm $f$ 's utility function determines a choice rule $C_{f}$ : for $A \subset X, C_{f}(A)$ is the maximal subset of $A$ according to $u_{f}$. Note that since $u_{f}$ is one-to-one, $C_{f}(A)$ is uniquely defined. The empty set $\emptyset$ represents for $f$ the option of hiring no workers. For notational convenience, I have not restricted the domain of $u_{f}$ to contracts with $f=x_{F}$, but of course we want $f$ to sign contracts only in its own name; assume then that $x \in C_{f}(A)$ implies $f=x_{F}$. Assume also that $x, x^{\prime} \in C_{f}(A)$ implies $x_{W} \neq x_{W}^{\prime}$.

For a worker $w, \emptyset$ represents an outside option: a contract that is always available to her if she chooses to reject the contract some firm offers her. Suppose that if $x_{W} \neq w$ then $u_{w}(x)<u_{w}(\emptyset)$.

A set of contracts $A$ is feasible if, for all workers $w$, there is at most one $x \in A$ with $w=x_{W}$.

A firm $f$ 's utility satisfies substitutability if, for any set of contracts $A$, and any two contracts $x$ and $x^{\prime}, x \notin C_{f}(A \cup\{x\})$ implies $x \notin C_{f}\left(A \cup\left\{x, x^{\prime}\right\}\right)$.

The tuple $\left(F, W, X,\left(u_{f}\right),\left(u_{w}\right)\right)$ describes a matching market with contracts.

A set of contracts $A \subset X$ is individually rational if, for all $x \in A, u_{x_{W}}(x) \geq u_{x_{W}}(\emptyset)$; and for all firms $f, C_{f}(A)=\left\{x \in A: f=x_{F}\right\}$.

A set of contracts $A \subset X$ is stable if it is individually rational and if for any firm $f$ and set of contracts $A^{\prime} \neq A$ with $A^{\prime}=C_{f}\left(A \cup A^{\prime}\right)$, there is one contract $x^{\prime} \in A^{\prime}$ such that either $u_{x_{W}^{\prime}}\left(x^{\prime}\right)<u_{x_{W}^{\prime}}(\emptyset)$ or there is $x \in A$ with $x_{W}=x_{W}^{\prime}$ and $u_{x_{W}^{\prime}}\left(x^{\prime}\right)<u_{x_{W}^{\prime}}(x)$. 
Salaries.-A matching market with salaries is described by:

- (finite, disjoint) sets $W$ of workers, $F$ of firms, and $S \subset \Re_{+}$of salaries;

- for each worker $w \in W$, a utility function $v_{w}: F \cup\{\emptyset\} \times S \rightarrow \Re$; and for each firm $f \in F$, a utility function $v_{f}: \cup_{A \subset W} A \times S \rightarrow \Re$; all utility functions are oneto-one (preferences are strict).

We can suppose that $S$ is the set $\{0,1, \ldots L\}$ of the first $L+1$ nonnegative integers, for some $L$.

For a firm $f, v_{f}$ defines a demand function $D_{f}: S^{W} \rightarrow 2^{W}$ by

$$
D_{f}(\mathbf{s})=\arg \max _{A \subset W} v_{f}\left(\left\{\left(w, s_{w}\right): w \in A\right\}\right) .
$$

I restrict attention to demand functions, not correspondences, by virtue of Hatfield and Milgrom's assumption of strict preferences. Say that $D$ satisfies gross substitutes if, for any two vectors of salaries, $\mathbf{s}$ and $\mathbf{s}^{\prime}$, if $\mathbf{s} \leq \mathbf{s}^{\prime}$ and $s_{w}=s_{w}^{\prime}$ then $w \in D(\mathbf{s})$ implies that $w \in D\left(\mathbf{s}^{\prime}\right)$.

A matching is a function $\mu: W \rightarrow F \cup\{\emptyset\} \times S$. A matching assigns to each worker a firm and a salary; I use the $\mu(w)=(\emptyset, 0)$ notation for when $w$ is unmatched (unemployed). A matching specifies for each firm $f$ a collection of workers with their corresponding salaries: $\mu^{0}(f)=\{(w, s):(f, s)=\mu(w)\}$. The set $\mu^{0}(f)$ is thus the set of workers employed by $f$, and their salaries, in the matching $\mu$.

The tuple $\left(F, W, S,\left(v_{f}\right),\left(v_{w}\right)\right)$ describes a matching market with salaries.

A matching $\mu$ is individually rational if, for every $f$ and $w, v_{f}\left(\mu^{0}(f)\right) \geq v_{f}(B)$ for all $B \subset \mu^{0}(f)$ and $v_{w}(\mu(w)) \geq v_{w}(\emptyset, 0)$.

A matching $\mu$ is stable if it is individually rational and if, for any firm $f$ and $A \subset W$, if there is a vector of salaries $\left(s_{w}\right)_{w \in A}$ with $v_{f}\left(\left\{\left(w, s_{w}\right): w \in A\right\}\right)>v_{f}\left(\mu^{0}(f)\right)$ then there is $w \in A$ with $v_{w}(\mu(w))>v_{w}\left(f, s_{w}\right)$.

\section{B. Embedding}

Let $\left(F, W, X,\left(u_{f}\right),\left(u_{w}\right)\right)$ be a matching market with contracts, and $\left(F, W, S,\left(v_{f}\right)\right.$, $\left.\left(v_{w}\right)\right)$ a matching market with salaries. An embedding of $\left(F, W, X,\left(u_{f}\right),\left(u_{w}\right)\right)$ into $\left(F, W, S,\left(v_{f}\right),\left(v_{w}\right)\right)$ is a one-to-one function $g$ which maps each $x \in X$ into a triple $\left(x_{F}, x_{W}, s\right) \in F \times W \times S$.

Let $g$ be such an embedding, and $A \subset X$. Say that $g(A)$ defines a matching if for any $w$ there is at most one $s$ and $f$ with $(f, w, s) \in g(A)$. The matching defined by $A$ under $g$ is the function $\mu: W \rightarrow F \times S$ defined by $\mu(w)=(f, s)$ if $g^{-1}(f, w, s) \in A$ and $\mu(w)=(\emptyset, 0)$ otherwise.

THEOREM 1: Let $\left(F, W, X,\left(u_{f}\right),\left(u_{w}\right)\right)$ be a matching market with contracts. If firms' choices satisfy substitutability, then there is a matching market with salaries $\left(F, W, S,\left(v_{f}\right),\left(v_{w}\right)\right)$, and an embedding $g$ of $\left(F, W, X,\left(u_{f}\right),\left(u_{w}\right)\right)$ into $\left(F, W, S,\left(v_{f}\right),\left(v_{w}\right)\right)$ such that

1) firms' demand functions in $\left(F, W, S,\left(v_{f}\right),\left(v_{w}\right)\right)$ satisfy gross substitutes;

2) $A \subset X$ is a set of stable contracts if and only if $g(A)$ defines a stable matching. 
The proof of Theorem 1 works by constructing an embedding. As suggested in the introduction, the Pareto frontier of contracts is "one dimensional": what is better for the worker is worse for the firm. So Pareto optimal contracts may be translated into salaries, with the better contracts for the firm defining lower salaries, and the better contracts for the worker defining higher salaries. The problem is that a firm's ranking over contracts might be affected by the firm's other hires. For example, consider contracts $x$ and $x^{\prime}$, both involving worker $w$ and firm $f$. Suppose that $x$ is better than $x^{\prime}$ for $f$, and $x^{\prime}$ is better than $x$ for $w$; so we would map $x$ into a lower salary than $x^{\prime}$. In the absence of substitutes, this mapping might be affected by the presence of other contracts. However, since $x^{\prime}$ is rejected by $f$ when $x$ is available, substitutes make sure that it will continue to be rejected when other workers and contracts are available. So the assumption of substitutes allows for the pairwise mapping of contracts into salaries to work globally, when all workers and firms are considered. The construction uses the salaries $1,2,3, \ldots$; but any other grid works in exactly the same way.

\section{PROOF:}

Say that a contract $x$ is dominated for $x_{F}$ and $x_{W}$ if there is a contract $x^{\prime}$ with $x_{F}$ $=x_{F}^{\prime}, x_{W}=x_{W}^{\prime}, u_{x_{F}}\left(x^{\prime}\right)>u_{x_{F}}(x)$ and $u_{x_{W}}\left(x^{\prime}\right)>u_{x_{W}}(x)$. Let $X_{f w}$ be the set of all contracts $x$ with $x_{F}=f$ and $x_{W}=w$ that are not dominated for $f$ and $w$. Note that $X_{f w}$ can be ordered by $u_{w}$; that is, I can enumerate the elements of $X_{f w}$ as $x_{1}, \ldots, x_{\left\|X_{f w}\right\|}$ with $u_{w}\left(x_{i}\right)<u_{w}\left(x_{i+1}\right)$. Then I can write $X_{f w}=\left\{(w, s): s=1, \ldots,\left\|X_{f w}\right\|\right\}$ with the understanding that $(w, s)$ corresponds to offering worker $w$ the contract $x_{s}$ in $X_{f w}$. Note that $s<s^{\prime}$ if and only if $u_{f}(\{w, s\})>u_{f}\left(\left\{w, s^{\prime}\right\}\right)$ : by definition, if $s<s^{\prime}$ then $u_{w}(f, s)<$ $u_{w}\left(f, s^{\prime}\right)$ so $u_{f}(\{w, s\})<u_{f}\left\{w, s^{\prime}\right\}$ would imply that $(w, s)$ is dominated.

Let $K=\max \left\{\left\|X_{f w}\right\|: f \in F, w \in W\right\}$ and $S=\{1,2, \ldots, K+1\}$. For convenience, let's augment the contracts in $X_{f w}$ to include $(w, s)$ with $\left\|X_{f w}\right\|<s \leq K+1$. Assume that if $s>\left\|X_{f w}\right\|$ then, for all $A,(w, s) \notin C_{f}(A)$. The embedding $g$ is the mapping that takes $x \in X$ into $(f, w, s)$ with $(w, s)$ being the representation of $x$ in $X_{f w}$ if $x$ is not dominated, and into $\left(x_{F}, x_{F}, K+1\right)$ if it is. ${ }^{2}$

Define firms' and workers' utilities as follows. Let $v_{w}(f, s)=u_{w}(x)$, where $x \in X_{f w}$ corresponds to $s$. Let $v_{f}\left(\left(w_{1}, s_{1}\right), \ldots,\left(w_{n}, s_{n}\right)\right)=u_{f}\left(\left(w_{1}, s_{1}\right), \ldots,\left(w_{n}, s_{n}\right)\right)$. For a vector of wages $\mathbf{s}=\left(s_{w}\right)_{w \in W} \in S^{W}$, let $X_{f}^{\mathbf{s}} \subset X_{f}$ be the set of contracts $\left(w, s_{w}\right)$. Define a demand function $D_{f}$ for firm $f$ by $D_{f}(\mathbf{s})=C_{f}\left(X_{f}^{\mathbf{s}}\right)$ (this is consistent with our definition of utility for $f$ ).

I shall now prove that $D_{f}$ satisfies the GS property. Let $X_{f}^{\mathbf{s}+}=\{(w, s) \in W \times S$ : $\left.s \geq s_{w}\right\}$, where $s_{w}$ denotes $w$ 's salary in $s$. I first prove that $C_{f}\left(X_{f}^{\mathbf{s}}\right)=C_{f}\left(X_{f}^{\mathbf{s}+}\right)$. If $X_{f}^{\mathbf{s}+}=X_{f}^{\mathrm{s}}$ then there is nothing to prove. Let $(w, s) \in X_{f}^{\mathbf{s}+} \backslash X_{f}^{\mathrm{s}}$. There must exist some $s^{\prime}$, with $s^{\prime}<s$ and $\left(w, s^{\prime}\right) \in X_{f}^{\mathbf{s}^{+}}$. Note that $s^{\prime}<s$ implies $(w, s) \notin C_{f}\left(\left\{(w, s),\left(w, s^{\prime}\right)\right\}\right)$ because $u_{f}(w, s)<u_{f}\left(w, s^{\prime}\right)$ and $C_{f}\left(\left\{(w, s),\left(w, s^{\prime}\right)\right\}\right)$ cannot contain two contracts with the same worker. So $\left\{(w, s),\left(w, s^{\prime}\right)\right\} \subset X_{f}^{\text {s+ }}$ implies, by the property of substitutability, that $(w, s) \notin C_{f}\left(X_{f}^{\mathbf{s}+}\right)$. Thus I have shown that $C_{f}\left(X_{f}^{\mathbf{s}+}\right) \subset C_{f}\left(X_{f}^{s}\right)$. Since $X_{f}^{\mathbf{s}} \subset$ $X_{f}^{\mathrm{s}+}$, the definition of $C_{f}$ implies that $C_{f}\left(X_{f}^{\mathrm{s}+}\right)=C_{f}\left(X_{f}^{\mathrm{s}}\right)$.

Now, let $\mathbf{s}=\left(s_{w}\right)$ and $\mathbf{s}^{\prime}=\left(s_{w}^{\prime}\right)$ be vectors with $\mathbf{s} \leq \mathbf{s}^{\prime}$ while for $w_{0} \in W$, $s_{w_{0}}=s_{w_{0}}^{\prime}$, and $w_{0} \in D_{f}(\mathbf{s})$. Suppose by way of contradiction that $w_{0} \notin D_{f}\left(\mathbf{s}^{\prime}\right)$. Then

\footnotetext{
${ }^{2}$ Strictly speaking, for dominated $x$ we would need to set $g(x)=\left(x_{F}, x_{F}, K+l\right)$, choosing $l \geq 1$ so that $g$ remains one-to-one.
} 
$\left(w_{0}, s_{w_{0}}^{\prime}\right) \notin C_{f}\left(X_{f}^{\mathbf{s}^{\prime}}\right)=C_{f}\left(X_{f}^{\mathbf{s}^{\prime}+}\right)$. But then $X_{f}^{\mathrm{s}^{\prime}}+\subset X_{f}^{\mathrm{s}+}$ so substitutability implies that $\left(w_{0}, s_{w_{0}}^{\prime}\right) \notin C_{f}\left(X_{f}^{\mathrm{s}+}\right)$. Now, $C_{f}\left(X_{f}^{\mathrm{s}+}\right)=C_{f}\left(X_{f}^{\mathbf{s}}\right)$ and $\left(w_{0}, s_{w_{0}}\right)=\left(w_{0}, s_{w_{0}}^{\prime}\right)$ implies that $\left(w_{0}, s_{w_{0}}\right) \notin C_{f}\left(X_{f}^{\mathbf{s}}\right)$, a contradiction.

The proof that $A$ is stable in $\left(F, W, X,\left(u_{f}\right),\left(u_{w}\right)\right)$ if and only if $g(A)$ is stable in $\left(F, W, S,\left(v_{f}\right),\left(v_{w}\right)\right)$ is straightforward.

\section{Discussion}

\section{A. Antecedents}

The identification of contracts and salaries is not new. Both Crawford and Knoer (1981), and Kelso and Crawford (1982), mention how salaries can be interpreted as contracts. Roth (1984), who presents an early model of matching with contracts, also identifies contracts with salaries. The new observation here is that substitutability is important for the identification of contracts and salaries. It is not mentioned in the literature that follows Hatfield and Milgrom (2005). ${ }^{3}$

\section{B. Quasilinearity}

In Kelso and Crawford, firms' profits are quasilinear, but their existence proof is more general and does not depend on quasilinearity. One detail is that they require a salary that is high enough so no worker would be hired at that salary (see their Lemma 2). In the embedding in the theorem, we do have such a salary.

\section{Bilateral Substitutes}

In a model of matching with contracts, Hatfield and Kojima (2010) present a generalization of substitutes, called bilateral substitutes. They show stable matching with contracts exists under bilateral substitutes. Here I show that the model of Hatfield-Kojima cannot be embedded into the Kelso-Crawford model.

The following example is from Hatfield and Kojima (2010). Let the set of firms be $\left\{f, f^{\prime}\right\}$, the set of workers $\left\{w, w^{\prime}\right\}$, and the set of contracts be $\left\{x, \tilde{x}, z, \tilde{z}, z^{\prime}\right\}$. Let $x$ and $\tilde{x}$ involve firm $f$ and worker $w$, while $z$ and $\tilde{z}$ involve worker $w^{\prime}$ and firm $f$. Contract $z^{\prime}$ is between $w^{\prime}$ and $f^{\prime}$. Suppose that agents' utilities are such that their preferences are:

\begin{tabular}{rrrr}
$\succcurlyeq_{f}$ & $\succcurlyeq_{f^{\prime}}$ & $\succcurlyeq_{w}$ & $\succcurlyeq_{w^{\prime}}$ \\
\hline$\{x, z\}$ & $\left\{z^{\prime}\right\}$ & $\tilde{x}$ & $z$ \\
$\{\tilde{z}\}$ & $\emptyset$ & $x$ & $z^{\prime}$ \\
$\{\tilde{x}\}$ & & $\emptyset$ & $\tilde{z}$ \\
$\{x\}$ & & & $\emptyset$ \\
$\{z\}$ & & & \\
$\emptyset$. & & &
\end{tabular}

\footnotetext{
${ }^{3}$ Recently, my result has been extended by Scott Kominers to the many-to-many matching model (see Kominers forthcoming).
} 
I have omitted the alternatives that are worse than $\emptyset$. Suppose that there is an embedding, where $x$ maps to the salary $s_{x}$ and $\tilde{x}$ to the salary $s_{\tilde{x}}$. Then $x \notin C_{f}(\{x, \tilde{x}\})$ means that $s_{x}>s_{\tilde{x}}$. This would imply that

$$
u_{f}\left(x_{W}, z_{W}, s_{x}, s_{z}\right)<u_{f}\left(\tilde{x}_{W}, z_{W}, s_{\tilde{x}}, s_{z}\right)
$$

as $x_{W}=\tilde{x}_{W}$, which is incompatible with the preferences above.

I should clarify that a deviation from substitutes does not by itself prevent the model with contracts from being embeddable into a model with salaries. The structure of bilateral substitutes is interesting because it is a deviation from substitutes for which existence is guaranteed.

\section{Algorithm}

Under the embedding, the algorithm proposed by Hatfield and Milgrom (2005) is equivalent to the algorithm proposed by Kelso and Crawford (1982). It is easy to see that they are equivalent because the two algorithms find the same matching: the firm-optimal or the worker-optimal matching, depending on how the algorithms are formulated.

Here, I want to show that the algorithms not only calculate the same matchings, but that they also work in essentially the same way: the algorithms take equivalent routes to a stable matching. The Hatfield-Milgrom algorithm starts by each firm $f$ "offering" the best contracts for $f$, and the workers sequentially rejecting offers. In the embedding, the best contracts correspond to the lowest salaries; and, in a similar vein, the Kelso-Crawford algorithm starts at the lowest salaries. In Hatfield-Milgrom, when a contract gets rejected, it is as if the salary for that worker is raised. Similarly, the Kelso-Crawford algorithm works by raising the salaries of workers who reject an offer. Broadly speaking, the steps taken by the Hatfield-Milgrom algorithm represent movements along the Pareto frontier of contracts-movements in a direction that corresponds to higher salaries in the Kelso-Crawford model.

The following example illustrates the point and, I hope, helps in understanding the nature of the embedding better.

Suppose there are two firms and two workers: $F=\left\{f_{1}, f_{2}\right\}$ and $W=\left\{w_{1}, w_{2}\right\}$. Let $X=\left\{x_{1}, x_{3}, x_{2}, y_{1}, y_{2}\right\}$ be the set of contracts.

The agents' preferences are:

\begin{tabular}{rrrr}
$\succcurlyeq_{f_{1}}$ & $\succcurlyeq_{f_{2}}$ & $\succcurlyeq_{w_{1}}$ & $\succcurlyeq_{w_{2}}$ \\
\hline$\left\{x_{1}\right\}$ & $\left\{y_{2}\right\}$ & $x_{3}$ & $y_{1}$ \\
$\left\{x_{2}\right\}$ & $\left\{x_{3}\right\}$ & $x_{2}$ & $\emptyset$ \\
$\left\{y_{1}\right\}$ & $\emptyset$ & $x_{1}$ & \\
$\emptyset$ & & $\emptyset$. &
\end{tabular}

The Hatfield-Milgrom algorithm takes the steps in the following table. I am using the same notation as Hatfield-Milgrom: $R_{W}$ and $R_{F}$ are the rejected contracts of, respectively, workers and firms. 


\begin{tabular}{lllll} 
& $X_{W}$ & $R_{W}\left(X_{W}\right)$ & $X_{F}$ & $R_{F}\left(X_{F}\right)$ \\
\hline 0) & $\emptyset$ & $\emptyset$ & $X$ & $\left\{x_{2}, y_{1}, x_{3}\right\}$ \\
1) $\left\{x_{1}, y_{2}\right\}$ & $\left\{y_{2}\right\}$ & $X$ & $\left\{x_{2}, y_{1}, x_{3}\right\}$ \\
2) $\left\{x_{1}, y_{2}\right\}$ & $\left\{y_{2}\right\}$ & $\left\{x_{1}, x_{2}, x_{3}, y_{1}\right\}$ & $\left\{x_{2}, y_{1}\right\}$ \\
3) $\left\{x_{1}, y_{2}, x_{3}\right\}$ & $\left\{x_{1}, y_{2}\right\}$ & $\left\{x_{1}, x_{2}, x_{3}, y_{1}\right\}$ & $\left\{x_{2}, y_{1}\right\}$ \\
4) $\left\{x_{1}, y_{2}, x_{3}\right\}$ & $\left\{x_{1}, y_{2}\right\}$ & $\left\{x_{2}, x_{3}, y_{1}\right\}$ & $\left\{y_{1}\right\}$ \\
5) $\left\{x_{1}, x_{2}, x_{3}, y_{2}\right\}$ & $\left\{x_{1}, x_{2}, y_{2}\right\}$ & $\left\{x_{2}, x_{3}, y_{1}\right\}$ & $\left\{y_{1}\right\}$ \\
6) $\left\{x_{1}, x_{2}, x_{3}, y_{2}\right\}$ & $\left\{x_{1}, x_{2}, y_{2}\right\}$ & $\left\{x_{3}, y_{1}\right\}$ & $\emptyset$ \\
7) $X$ & $X$ & $\left\{x_{1}, x_{2}, y_{2}\right\}$ & $\left\{x_{3}, y_{1}\right\}$ & $\emptyset$
\end{tabular}

I shall interpret the iterations so as to simplify the comparison with KelsoCrawford. In (0) firms "offer" contracts $x_{1}$ and $y_{2}$ (they reject contracts $\left\{x_{2}, y_{1}, x_{3}\right\}$. In 1) the workers respond by $w_{1}$ temporarily accepting $x_{1}$, while $w_{2}$ rejects $y_{2}$. In 2 ) the firms offer $x_{1}$ and $x_{3}$. In 3) $w_{1}$ accepts $x_{3}$ and rejects $x_{2}$. In 4) $f_{1}$ offers $x_{2}$ and $f_{2}$ offers $x_{3}$. 5) $w_{1}$ rejects $x_{2}$. 6) $f_{1}$ offers $y_{1}$ and $f_{2}$ offers $x_{3}$. 7) the workers accept $y_{1}$ and $x_{3}$.

We can embed this market with contracts into a market with salaries. Let the set of salaries be $\{1,2\}$. The relevant part of the agents' preferences are:

$$
\begin{aligned}
& f_{1}:\left(w_{1}, 1\right) \succ\left(w_{1}, 2\right) \succ\left(w_{2}, 1\right) \\
& f_{2}:\left(w_{2}, 1\right) \succ\left(w_{1}, 1\right) \\
& w_{1}:\left(f_{2}, 1\right) \succ\left(f_{1}, 2\right) \succ\left(f_{1}, 1\right) \\
& w_{2}:\left(f_{1}, 1\right)
\end{aligned}
$$

So, firm $f_{1}$ prefers to hire $w_{1}$ at a lower rather than a higher salary, and prefers to hire $w_{1}$ at either salary over hiring $w_{2}$ at a salary of 1 . I omit, for example, that for $w_{1}\left(f_{2}, 2\right) \succ\left(f_{2}, 1\right)$ because firm $f_{2}$ is unwilling to hire $w_{1}$ at a salary of 2 (she prefers to leave the position vacant).

Kelso and Crawford's algorithm does:

\begin{tabular}{lll}
$w_{1}$ & $w_{2}$ \\
\hline 0) & $\underline{\left(f_{1}, 1\right)}$ & $\left(f_{2}, 1\right)$ \\
1) & $\left(f_{1}, 1\right) \underline{\left(f_{2}, 1\right)}$ & \\
2) & $\left(f_{1}, 2\right) \underline{\left(f_{2}, 1\right)}$ & \\
3) & $\underline{\left(f_{2}, 1\right)}$ & $\underline{\left(f_{1}, 1\right)}$.
\end{tabular}

The meaning of the iterations is the following. The algorithm starts with all salaries being 1 . At this vector of salaries, $f_{1}$ demands worker $w_{1}$ while $f_{2}$ demands worker $w_{2}$. Worker $w_{2}$ rejects the offer of firm 2 while $w_{1}$ accepts the offer from $f_{1}$; this is indicated by underlining the offer in the table. Since $f_{2}$ 's offer to $w_{2}$ was rejected, the salary for that pair increases to 2 . At that salary, $f_{2}$ decides not to offer to $w_{2}$ and instead demand $w_{1}$ at a salary of 1 . The rest of the iterations should be intuitive. In any case, the steps are essentially those of the Hatfield-Milgrom algorithm, where workers' responses are included within each step in the Kelso-Crawford algorithm, and as a separate step in Hatfield-Milgrom. A general equivalence result is possible 
but requires writing down an algorithm that interprets the steps of one algorithm in terms of the other.

\section{E. Law of Demand}

In a matching model with contracts, a firm $f$ satisfies the law of aggregate demand if $A \subseteq A^{\prime}$ implies $\left\|C_{f}(A)\right\| \leq\left\|C_{f}\left(A^{\prime}\right)\right\|$. In a matching model with salaries, a firm $f$ satisfies the law of aggregate demand if $\mathbf{s} \geq \mathbf{s}^{\prime}$ implies that $\left\|D_{f}\left(\mathbf{s}^{\prime}\right)\right\| \geq\left\|D_{f}(\mathbf{s})\right\|$.

Using the embedding of Theorem 1, it is easy to show that if a firm satisfies the law of aggregate demand in the matching model with contracts, then it satisfies the law of aggregate demand in the embedded matching model with salaries.

\section{REFERENCES}

Crawford, Vincent P., and Elsie Marie Knoer. 1981. "Job Matching with Heterogeneous Firms and Workers." Econometrica 49(2): 437-50.

Hatfield, John William, and Fuhito Kojima. 2010. "Substitutes and Stability for Matching with Contracts." Journal of Economic Theory 145(5): 1704-23.

Hatfield, John William, and Paul R. Milgrom. 2005. "Matching with Contracts." American Economic Review 95(4): 913-35.

Kelso, Alexander S., Jr., and Vincent P. Crawford. 1982. "Job Matching, Coalition Formation, and Gross Substitutes.” Econometrica 50(6): 1483-1504.

Kominers, Scott. Forthcoming. "On the Correspondence of Contracts to Salaries in (Many-to-Many) Matchings." Games and Economic Behavior.

Roth, Alvin E. 1984. "Stability and Polarization of Interests in Job Matching." Econometrica 52(1): 47-57.

Roth, Alvin E., and Marilda A. Oliveira Sotomayor. 1990. Two-Sided Matching: A Study in GameTheoretic Modeling and Analysis. New York: Cambridge University Press. 
This article has been cited by:

1. Christian Ahlin. 2017. Matching Patterns When Group Size Exceeds Two. American Economic Journal: Microeconomics 9:1, 352-384. [Abstract] [View PDF article] [PDF with links] 